\title{
Correction to: Sodium-Glucose Cotransporter 2 Inhibitors for Prevention of Heart Failure Events in Patients with Type 2 Diabetes Mellitus: A Cost Per Outcome Analysis
}

\author{
Ronen Arbel ${ }^{1}\left[\right.$. Enis Aboalhasan ${ }^{1} \cdot$ Ariel Hammerman $^{2} \cdot$ Joseph Azuri $^{3,4}$
}

Published online: 6 June 2020

(c) Springer Nature Switzerland AG 2020

\section{Correction to: Clinical Drug Investigation https://doi.org/10.1007/s40261-020-00929-z}

The original version of this article unfortunately contained a mistake. The correct information is given below.

Abstract—Results, 1st sentence, which previously read:

We figured empagliflozin's CNT to be $\$ 664,464$ (95\% CI $\$ 499,872-\$ 1,097,280)$ ), $\$ 1,535,387$ (95\% CI $\$ 886,074-$ $\$ 3,210,501)$ for canagliflozin, and $\$ 2,693,145$ (95\% CI $\$ 1,639,563-\$ 11,092,206)$ for dapagliflozin.

Should read:

We figured empagliflozin's CNT to be $\$ 664,464$ (95\% CI $\$ 499,872-\$ 1,097,280)$, $\$ 1,353,387$ (95\% CI $\$ 886,074-$ $\$ 3,210,501)$ for canagliflozin, and $\$ 2,693,145$ (95\% CI $\$ 1,639,563-\$ 11,092,206$ ) for dapagliflozin.

The original article can be found online at https://doi.org/10.1007/ s40261-020-00929-z.

Ronen Arbel

ronen.arbel@gmail.com

1 Maximizing Health Outcomes Research Lab, Department of Technology Marketing, Sapir College, D.N. Hof Ashkelon, 79165 Sderot, Israel

2 Department of Pharmaceutical Technology Assessment, Clalit Health Services Headquarters, Tel-Aviv, Israel

3 Diabetes Clinic, Central District, Maccabi Healthcare Services, Tel Aviv, Israel

4 Sackler Faculty of Medicine, Tel Aviv University, Tel Aviv, Israel 